\title{
The pain of procedural pain management education in the emergency department
}

\author{
Ran D. Goldman, MD, MH(Research)*
}

Emergency medicine (EM) and pediatric emergency medicine (PEM) in Canada have come a long way since Swafford and Allen suggested in 1968 that "pediatric patients seldom need medication for the relief of pain. They tolerate discomfort well." "1 Yet, in 2005, Johnston et al. $^{2}$ from Montreal reported that in two urban university-affiliated pediatric emergency departments (PEDs), only a small proportion of children received analgesia during a visit to the emergency department (ED) and their distress lasted at least 1 week after discharge. Also, in 2014, Kircher et al. ${ }^{3}$ reported in CFEM that pain scores of children with musculoskeletal injuries were recorded for only $6 \%$ of patients in two EDs in Alberta, and the average time from triage to the first analgesic in the ED was on average as long as 2 hours.

In this journal of CFEM, Poonai et al. ${ }^{4}$ report the results of a survey among pediatric emergency physicians (EPs) and general EPs showing abridged willingness to provide analgesia to a 3 -week-old infant for a lumbar puncture procedure, compared with a 16-year-old or a 3 -year-old child, likely due to unfamiliarity surrounding analgesic options and the perception that analgesia will produce additional discomfort to the neonate.

These findings are similar to a survey of Canadian pediatrics and general EM residents from a decade ago, ${ }^{5}$ in which lumbar punctures were reported to be performed with no local anesthetics or sedation, and a U.S.-based survey reporting that two-thirds of pediatricians do not routinely use analgesia for neonatal lumbar punctures. ${ }^{6}$ Nonetheless, the new findings are disheartening, and, despite educational efforts over the decades, members of the EM and PEM specialties continue to hold perceptions and misconceptions when it comes to neonatal pain.
It is even possible that reported practices by these EM and PEM providers are an underestimate of true practice. An abysmal 24\% of children received some form of pain management before their lumbar puncture in a 2010 report from New York. ${ }^{7}$ An even more embarrassing $6.5 \%$ of infants 0-2 months old and 14\% of infants 3-18 months of age received any analgesia, with younger patients receiving significantly less frequent pain management $(p<0.001)$.

These recent findings should not surprise us, because there is likely something fundamental that is not working. When it comes to education of pain competencies, veterinary medicine in Canada has a much more robust pain management curriculum than medicine, nursing, and dentistry. ${ }^{8}$ Graduates of veterinary medicine have such a comprehensive list of competencies related to pain, that they include one that is explicitly related to the responsibility to "alleviate suffering" in an emergency situation. ${ }^{8}$ Furthermore, pediatric residents that were responsible for teaching trainees how to perform a lumbar puncture received minimal educational opportunities themselves during residency, leading them to be reluctant to recommend the use of local anesthetics when teaching the procedure. ${ }^{5}$

Nonetheless, before us is a newfangled opportunity that should not be missed. Competence by Design (CBD), a hybrid, outcomes-based approach to training and assessment, planned as a flexible and adaptable learning program, may overcome current gaps in pediatric pain management knowledge and perception, and can support training and lifelong learning for generations of acute care providers.

In the new CBD of anesthesiologists, "managing uncomplicated patients with acute pain" serves as an

From the *Divisions of Pediatric Emergency Medicine and Pediatric Clinical Pharmacology, Department of Pediatrics, BC Children's Hospital and BC Children's Hospital Research Institute, University of British Columbia, Vancouver, BC.

Correspondence to: Dr. Ran D. Goldman, BC Children's Hospital, 4480 Oak St., Vancouver, BC V6H 3N1; Email: rgoldman@cw.bc.ca 
example for an entrustable professional activity (EPA) that residents must attain in order to move to the next stage of training. The newly minted EM CBD and the future PEM CBD (anticipated after 2020) programs must include pediatric acute procedural pain management capabilities as part of their core competencies.

As EM and PEM providers, we are required to remember that successful CBD must rely on us, by ensuring well-defined objectives for learning, focus on personal resident development, more frequent assessments, and meaningful feedback to our trainees. It is in our young patients' best interest that we include practical clinical pain education as part of preparation for independent practice in EM and PEM.

When it comes to analgesia, discrepancies continue to exist between reported knowledge and beliefs of medical staff, and their clinical practice. ${ }^{9}$ It is in our hands to embrace this opportunity to teach appropriate and evidence-based management of pain and suffering during procedures in the ED.

Keywords: Analgesia, emergency services, pain, pediatric emergency department, procedures

Competing interests: None declared.

\section{REFERENCES}

1. Swafford LI, Allen D. Pain relief in the pediatric patient. Med Clin North Am 1968;52:131-6.

2. Johnston CC, Bournaki MC, Gagnon AJ, et al. Self-reported pain intensity and associated distress in children aged 4-18 years on admission, discharge, and one-week follow up to emergency department. Pediatr Emerg Care 2005;21(5):342-6.

3. Kircher J, Drendel AL, Newton AS, et al. Pediatric musculoskeletal pain in the emergency department: a medical record review of practice variation. CFEM 2014;16(6):449-57.

4. Poonai N, Brzozowski V, Stang AS, et al. Pain management practices surrounding lumbar punctures in children: A survey of Canadian emergency physicians. C7EM 2019;21(2):199-203.

5. Breakey VR, Pirie J, Goldman RD. Pediatric and emergency medicine residents' attitudes and practices for analgesia and sedation during lumbar puncture in pediatric patients. Pediatrics 2007;119(3):e631-6.

6. Baxter AL, Welch JC, Burke BL, Isaacman DJ. Pain, position, and stylet styles: infant lumbar puncture practices of pediatric emergency attending physicians. Pediatr Emerg Care 2004;20(12):816-20.

7. Fein DF, Avner JR, Khine H. Pattern of pain management during lumbar puncture in children. Pediatr Emerg Care 2010;26:357-60.

8. Watt-Watson J, Peter E, Clark AJ, et al. The ethics of Canadian entry-to-practice pain competencies: how are we doing? Pain Res Manag 2013;18(1):25-32.

9. Jacob E, Puntillo KA. Variability of analgesic practices for hospitalized children on different pediatric specialty units. 7 Pain Symptom Manage 2000;20:59-67. 